\title{
A Crucial Part of the Conversation in Education: Receiving Feedback
}

\author{
Thanks for the feedback. The science and art of receiving feedback well. By Douglas Stone and \\ Sheila Heen; Penguin Books; New York, New York; 2014; ISBN 9780143127130; pp 348; \$ 18 \\ (paperback)
}

\author{
Richard Balon ${ }^{1}$ (B)
}

Received: 9 November 2021 / Accepted: 7 January 2022 / Published online: 27 January 2022

(C) Academic Psychiatry 2022

"We swim in an ocean of feedback." (p. 1)

"Feedback includes any information you get about yourself. In the broader sense, it's how we learn about ourselves from our experiences and from other people - how we learn from life." (p. 4)

Providing and receiving feedback is a crucial part of all education. Feedback provides trainees, and at times teachers, with valuable information about their skills, knowledge, behavior, professionalism, and other aspects of performance. We, as teachers, have been somewhat preoccupied with delivering feedback the "right way." We try to improve our skills in delivering it, such as by applying the "sandwich method" (a piece of negative feedback sandwiched between two positive messages). However, it seems that we have paid less attention to the way in which we and our trainees receive feedback. In a way, giving and receiving feedback are two sides of the same coin, and if either side is not done appropriately, the currency of feedback is not valuable. Thus, we need to be able to deliver feedback in an appropriate way but also make sure that feedback is received and understood. Typically, we perceive that giving feedback is a skill, but receiving feedback is also a skill and both must be learned.

In their popular book Thanks for the Feedback, Douglas Stone and Sheila Heen, both lecturers at Harvard Law School, co-founders of the Triad Consulting Group, and members of the Harvard Negotiation Project think tank, are taking "an honest look at why receiving feedback is hard, and to provide a framework and some tools that can help you metabolize

Richard Balon

rbalon@wayne.edu

1 Wayne State University, Detroit, MI, USA challenging, ever crazy-making information and use it to fuel insight and growth" (p. 2). In the introduction, they describe that when people are asked about "their most difficult conversations, feedback always comes up," and "rarely goes well," as the receivers of feedback are upset, defensive, and lack motivation for change (p. 3). The authors note that "When we give feedback, we notice that the receiver isn't good at receiving it," and "When we receive feedback, we notice that the giver isn't good at giving it" (p. 3). In their analyses they finally conclude that "the key player" in feedback "is not the giver, but the receiver" (p. 3).

The introduction continues to provide more salient information on feedback. The authors write, "Feedback is not just what gets ranked; it's what gets thanked, commented on, and invited back, or dropped. Feedback can be formal or informal, direct or implicit; it can be blunt or baroque, totally obvious or so subtle that you're not sure what it is" (p. 4). They also remind us that "feedback plays a crucial role in developing talent, improving morale, aligning teams, solving problems, and boosting the bottom line" (p. 5). Yet, many people frequently feel that feedback is unfair or inaccurate, and they dread it. Thus, the authors feel that training in providing feedback can be helpful; however, "if the receiver isn't willing or able to absorb the feedback" (p. 5), it cannot be successful. Thus, they feel the emphasis should be on the receiver of feedback. They point out that the expectation to learn mainly from exceptional teachers and mentors is unrealistic, as those are rare, and thus we need to "learn from just about everyone" (p. 6). The authors also emphasize that learning about ourselves can be painful and that there is a lot of tension between learning about oneself and being accepted. I liked their question. "Is it possible that feedback is like a gift and like a colonoscopy? ... Is the learning really worth the pain?" (p. 8). They add, "Receiving feedback sits at the intersection of these two needs - our drive to learn and our longing for acceptance" (p. 8). In the last part of the Introduction, the 
authors write that feedback has a lot of benefits and that feedback-seeking behavior has been linked, for instance, to higher job satisfaction, greater creativity, and faster adaptation. They also emphasize that feedback does not always have to be taken and that receiving it well means skillful engagement in conversation with the giver.

Following the introduction, the book consists of two parts: "The Feedback Challenge" and "Feedback in Conversation." The discussion of challenge to feedback starts with a chapter on three triggers (basically obstacles) that block feedback (the authors point out that triggers are not just obstacles but also information): truth triggers (feedback is somehow off, unhelpful, or untrue); relationship triggers (related to both the giver and the receiver, as feedback is colored by the relationship between them and at times the focus shifts from the feedback itself to the person delivering it); and identity triggers (which are solely about "us" receivers, our feelings of being threatened or off balance, etc.). The discussion of truth triggers stresses the importance of understanding feedback in its many forms (appreciation? coaching? evaluation?). The way to get out of relationship triggers is to "disentangle the feedback from the relationship issues it triggers, and to discuss both, clearly and separately" (p. 21). The part on identity triggers makes a point about cultivating and growing one's identity, in order to better give and receive feedback.

The following eight chapters cover triggers in more detail. Truth triggers are discussed in three chapters: the first one on separating three kinds of feedback (appreciation, coaching, and evaluation); the second one on understanding what the trigger is and means and moving from "what is wrong" to "tell me more"; and the third one on seeing one's blind spots and discovering how one comes across.

In the discussion on separating appreciation, coaching, and evaluation, I liked some comments on appreciation, for example, that it has to be specific, reflective of receiver's values, and meaningful. The chapter also provides advice on what helps with specific triggers, such as getting purposes aligned and separating evaluation from coaching and appreciation. The chapter on understanding feedback suggests that frank discussion of content is very important. The following chapter on seeing one's blind spots and discovering how one comes across is quite interesting. It lists behavioral blind spots: "leaky face" (everyone but you can see your face); "leaky tone" (which conveys a lot of information about our feelings); "e-mail body language" (e.g., using all capital letters and lots of exclamation points and question marks, word choices, the timing of sending messages); and blind spot amplifiers (e.g., emotional; situation vs. character; or impact vs. intent), and what helps us to see our blind spots (e.g., looking for patterns, getting a second opinion, recording ourselves, focusing on change from the inside out).

The part on relationship triggers focuses on disentangling what from who (e.g., avoid "switchtracking," such as by saying "you do not like me" instead of "you are not listening to me") and on identifying relationship systems. The authors emphasize that switching tracks defeats feedback and that we can be "triggered" by who is giving the feedback. (Are they credible? Do we trust them?) The analysis of relationship systems notes, among others, that "accidental adversaries are created by two things: role confusion and role clarity" (p. 130) and advises to "take three steps back" to see the big picture that includes, for instance, other players, environment, policies, and timing.

The final part of "The Feedback Challenge" section reviews identity triggers in chapters on learning how wiring and temperament affect our story (e.g., our emotions have profound influence on how we interpret feedback) and on how to dismantle distortions in five ways: be prepared and be mindful, separate the strands: feeling/story/feedback, contain the story, change your vantage point, and accept that you can't control how others see you; and on how one needs to cultivate a growth identity. This section is full of useful advice, such as that we have to accept that we will make mistakes, we have complex intentions, and we contribute to the problem.

The second part of the book, related to "Feedback in Conversation," includes four chapters: how good does one have to be; navigating the conversation on feedback; how to get going and take action in five ways; and how to pull together things regarding feedback in organizations. The "how good does one have to be" chapter discusses finding and setting boundaries around receiving feedback and establishing limits on feedback. It suggests that it is okay not only to turn down feedback but also to say, "I don't even want to hear it" (p. 209). Saying no could be crucial to one's well-being. The authors state that being able to say no is right at the heart of receiving feedback. "If you can't say no, then your yeses are not freely chosen" (p. 209). The three boundaries to set: (1) "I may not take your advice"; (2) "I don't want feedback about that subject, not right now"; and (3) "Stop or I will leave the relationship." The text also includes suggestions on how one knows if boundaries are needed and where boundaries could help to improve feedback. Finally, the authors recommend that one turns away feedback with grace and honesty and is transparent, firm, and very appreciative and specific about requests and consequences.

The chapter on navigating the conversation recommends, among others, to close the feedback conversation with commitment. Finally, the chapter on getting going presents five ways to take action, such as naming one thing (e.g., asking "what's one thing you see me doing that gets in my own way?" [p. 258]); trying small experiments; riding out the "J curve" (i.e., plotting well-being and time on a graph); "coaching your coach" (which is difficult when the coach is your boss); and inviting in the feedback giver (i.e., a good listener asks for help). 
While reading this book, one realizes that feedback is more complex than one might think. It allows reflection on an important part of what we do as educators. We clearly need to change our culture of feedback and to focus also on how to receive it. This book underscores the need to educate ourselves and our trainees about both giving and receiving feedback, especially the negative (or "constructive") kind. In my experience, many trainees just want to hear that they do everything well, and faculty members are frequently good in delivering this message. Most people are hesitant to deliver what is considered "negative" feedback, and trainees frequently do not know how to receive it, not realizing the triggers of receiving feedback (e.g., getting very emotional) and that "negative" feedback could be useful. I think that if we cannot deliver feedback appropriately and if our trainees cannot learn how to receive feedback, the system is broken, and we are failing our trainees. This book provides readers with guidance on how to make sure our feedback is not only delivered well but also received well and in a useful manner. It would be helpful to use this book in discussions on feedback with trainees, explaining its ways, techniques, and significance; how they should handle it; and what they should do with it.

\section{Declarations}

Disclosures The author states that there is no conflict of interest.

Publisher's Note Springer Nature remains neutral with regard to jurisdictional claims in published maps and institutional affiliations. 